\title{
A DICHOTOMY LAW FOR THE DIOPHANTINE PROPERTIES IN $\beta$-DYNAMICAL SYSTEMS
}

\author{
MICHAEL COONS, MUMTAZ HUSSAIN, AND BAO-WEI WANG
}

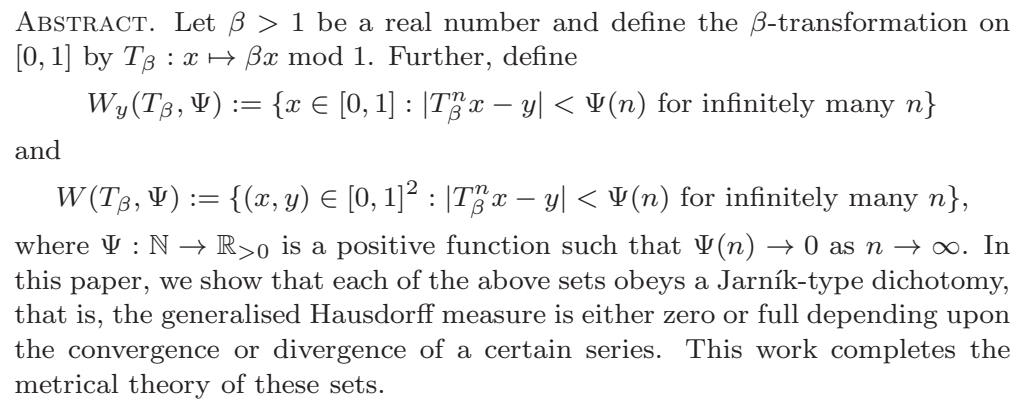

\section{INTRODUCTION}

Let $(X, T, \mu, \mathcal{B})$ be a measure-theoretic dynamical system, where $T: X \rightarrow X$ is a transformation on $X, \mu$ is a finite $T$-invariant Borel measure, and $\mathcal{B}$ is the associated Borel $\sigma$-algebra. The famous Poincaré recurrence theorem implies that for almost all $x \in X$, the $T$-orbit of $x$ is dense in $X$. That result is qualitative in nature, though it leads to the study of the quantitative properties of the distribution of the $T$-orbits of points in the space $X$, which is called dynamical Diophantine approximation. More precisely, the spotlight is on the size of the set

$$
W_{y}(T, \Psi):=\left\{x \in X:\left|T^{n} x-y\right|<\Psi(n) \text { for infinitely many } n\right\},
$$

where $\Psi: \mathbb{N} \rightarrow \mathbb{R}_{>0}$ is a positive function such that $\Psi(n) \rightarrow 0$ as $n \rightarrow \infty$. The set $W_{y}(T, \Psi)$ is the dynamical analogue of the classical well-approximable set (e.g., see [1, 2, 11, 22]) and it has close connections to classic Diophantine approximation, for example when $T$ is an irrational rotation or Gauss transformation. It has been an object of significant interest since the pioneering works of Philipp 28] on the $\mu$-measure of $W_{y}(T, \Psi)$ and Hill and Velani [17] on the Hausdorff dimension of $W_{y}(T, \Psi)$. It is easy to see from the definition that the set $W_{y}(T, \Psi)$ contains the points in $X$ whose $T$-orbit hits a shrinking target infinitely often; shrinking target problems for similar situations have been studied by Chernov and Kleinbock [10, Hill and Velani [17, 18, and Tseng [34 among others.

When the system $(X, T, \mu, \mathcal{B})$ possesses strong mixing properties, similar to Khintchine's theorem and its generalisations in classical Diophantine approximation, the $\mu$-measure of $W_{y}(T, \Psi)$ is zero or full, according to the convergence or divergence of a certain series. Philipp [28] proved this for $b$-ary expansions, $\beta$ expansions, and continued fractions.

2010 Mathematics Subject Classification. Primary 11J83; Secondary 11K60, 11K16.

Key words and phrases. Beta-expansions, Diophantine approximation, Jarník-type theorem, Hausdorff measure.

The research of M. Coons and M. Hussain is supported by the Australian Research Council (DE140100223) and the research of B.-W. Wang is supported by NSFC of China (No. 11471130 and NCET-13-0236). 
Properties of $W_{y}(T, \Psi)$ are related to the distribution of the inverse images $\left\{T^{-n} y\right\}_{n \geqslant 1}$ of $y$. If the $\mu$-measure of $W_{y}(T, \Psi)$ obeys a dichotomy law, it means, in some sense, that $\left\{T^{-n} y\right\}_{n \geqslant 1}$ is regularly distributed. In this way, one expects that tools from the theory of metric Diophantine approximation, such as regular systems [3, ubiquitous systems [7, 12, and the mass transference principle [5], can be used to derive the size of $W_{y}(T, \Psi)$ in terms of Hausdorff measure. More precisely, similar to the Jarník dichotomy law, one expects that there should be a dichotomy law (zero or full) for the Hausdorff measure of the dynamically defined limsup set $W_{y}(T, \Psi)$.

Following the work of Hill and Velani [17, 18, the Hausdorff dimension of the set $W_{y}(T, \Psi)$ has been determined in many systems, from the system of rational expanding maps on their Julia sets to systems with non-finite Markov systems 32 and conformal iterated function systems [25, 29, 35. However, the Hausdorff measure of $W_{y}(T, \Psi)$ is currently known only for systems with finite Markov properties [4, 19. We remedy this situation.

In this paper, we consider the Hausdorff measure of $W_{y}(T, \Psi)$ on $\beta$-expansions. There are two reasons that we choose to consider this non-finite Markov system. On the first hand, combining with Philipp's work, we hope to provide a complete metric theory on the size of $W_{y}(T, \Psi)$. Moreover, the non-finite Markov property for $\beta$-expansions remains a barrier to determining metric properties, so we want to see whether new ideas will be found in considering this concrete question. On the other hand, when given a full Lebesgue measure statement, the mass transference principle has proven a powerful tool in studying the Hausdorff measure of a limsup set in classic Diophantine approximation [5] as well as dynamical Diophantine approximation for systems with finite Morkov properties [4. But to the authors' knowledge, it seems that there are exceptions. For example, there is a full Lebesgue measure statement [24] on the size of the limsup set

$$
\{y:|n \alpha-y|<\psi(n) \text { for infinitely many } n \in \mathbb{N}\},
$$

but we do not think a direct application of mass transference principle would give even the right Hausdorff dimension, let alone its Hausdorff measure. For this nonfinite Markov system, we have to give some modifications on the mass transference principle and also need to carefully choose a subset of $W_{y}(T, \Psi)$ to make the mass transference principle applicable.

Now let's focus on the $\beta$-expansion. For a real number $\beta>1$, define the transformation $T_{\beta}:[0,1] \rightarrow[0,1]$ by

$$
T_{\beta}: x \mapsto \beta x \bmod 1 .
$$

This map generates the $\beta$-dynamical system $\left([0,1], T_{\beta}\right)$. It is well known that $\beta$ expansion is a typical example of an expanding non-finite Markov system whose properties are reflected by the orbit of some critical point; here, it is the expansion of 1 . General $\beta$-expansions have been widely studied in the literature, beginning with the pioneering works of Rényi [30, and Parry 26, and continuing with Hofbauer [20], Persson and Schmeling [27], Schmeling [31, and Tan and Wang [33] to name just a few.

We are interested in the size of the dynamically defined limsup set

$$
W_{y}\left(T_{\beta}, \Psi\right):=\left\{x \in[0,1]:\left|T_{\beta}^{n} x-y\right|<\Psi(n) \text { for infinitely many } n\right\},
$$

where, as above, $\Psi: \mathbb{N} \rightarrow \mathbb{R}_{>0}$ is a positive function. Philipp [28] showed that the Lebesgue measure or Parry measure of the set $W_{y}\left(T_{\beta}, \Psi\right)$ is zero or full according to the convergence or divergence of the series $\sum_{n \geqslant 1} \Psi(n)$. The Hausdorff dimension of $W_{y}\left(T_{\beta}, \Psi\right)$ was given by Shen and Wang [32] (see also Bugeaud and Wang [9]). As stated above, in this paper, we focus on the Hausdorff measure of $W_{y}\left(T_{\beta}, \Psi\right)$. 
Throughout this paper, a dimension function is a function $f: \mathbb{R} \rightarrow \mathbb{R}$ such that $f(r) \rightarrow 0$ as $r \rightarrow 0$ and such that $f$ is increasing in $\left[0, r_{0}\right)$ for some $r_{0}>0$; $\mathcal{H}^{f}$ denotes the $f$-dimensional Hausdorff measure. For the definitions of Hausdorff dimension and Hausdorff measure, we refer to the standard texts by Bernik and Dodson [7] and Falconer [13. In this paper, we prove the following dichotomy law for $\beta$-dynamical systems, which is an analogue of classical Jarník-type theorems. It is worth noting that our results are the first concerning Hausdorff measures of $\beta$-dynamical systems.

Theorem 1. Let $\Psi: \mathbb{N} \rightarrow \mathbb{R}_{>0}$ be a positive function. Let $f$ be a dimension function such that $r^{-1} f(r)$ is monotonic. For any $\beta>1$, we have

$$
\mathcal{H}^{f}\left(W_{y}\left(T_{\beta}, \Psi\right)\right)= \begin{cases}0 & \text { when } \sum_{n \geqslant 1} f\left(\frac{\Psi(n)}{\beta^{n}}\right) \beta^{n} \text { converges, } \\ \mathcal{H}^{f}([0,1]) & \text { when } \sum_{n \geqslant 1} f\left(\frac{\Psi(n)}{\beta^{n}}\right) \beta^{n} \text { diverges. }\end{cases}
$$

The condition ' $r^{-1} f(r)$ is monotonic' is not a particularly restrictive condition, and it is the main ingredient in unifying both the Lebesgue and Hausdorff measure statements; for details see Beresnevich and Velani [5]. To be precise, $\mathcal{H}^{f}$ is proportional to the standard Lebesgue measure when $f(r) \asymp r^{1}$. When $f(r) \asymp r^{s}$, we write $\mathcal{H}^{s}$ in place of $\mathcal{H}^{f}$, and whenever $\Psi(r)=r^{-\tau}$ for $\tau>0$, we write $W_{y}\left(T_{\beta}, \tau\right)$ in place of $W_{y}\left(T_{\beta}, \Psi\right)$.

Theorem 1 can be further generalised by considering the set

$$
W\left(T_{\beta}, \Psi\right):=\left\{(x, y) \in[0,1]^{2}:\left|T_{\beta}^{n} x-y\right|<\Psi(n) \text { for infinitely many } n\right\} .
$$

This set can be viewed as the doubly metrical $\beta$-dynamical analogue of the classic Diophantine set as given by Dodson [11. The Hausdorff dimension of $W\left(T_{\beta}, \Psi\right)$ was given by Ge and Lü [15]. Its Hausdorff measure is given as follows.

Theorem 2. Let $\Psi: \mathbb{N} \rightarrow \mathbb{R}_{>0}$ be a positive function. Let $g$ be a dimension function such that $r^{-2} g(r)$ is monotonic. For any $\beta>1$, we have

$$
\mathcal{H}^{g}\left(W\left(T_{\beta}, \Psi\right)\right)= \begin{cases}0 & \text { when } \sum_{n \geqslant 1} g\left(\frac{\Psi(n)}{\beta^{n}}\right) \frac{\beta^{2 n}}{\Psi(n)} \text { converges, } \\ \mathcal{H}^{g}\left([0,1]^{2}\right) & \text { when } \sum_{n \geqslant 1} g\left(\frac{\Psi(n)}{\beta^{n}}\right) \frac{\beta^{2 n}}{\Psi(n)} \text { diverges. }\end{cases}
$$

An immediate consequences of Theorems 1 and 2 are not only the respective Hausdorff dimension results, but also that $\mathcal{H}^{s}\left(W_{y}\left(T_{\beta}, \tau\right)\right)=\mathcal{H}^{s}([0,1])$ when $s=\operatorname{dim}_{H} W_{y}\left(T_{\beta}, \tau\right)=1 /(\tau+1)$, and $\mathcal{H}^{s}\left(W\left(T_{\beta}, \tau\right)\right)=\mathcal{H}^{s}\left([0,1]^{2}\right)$ when $s=$ $\operatorname{dim}_{H} W\left(T_{\beta}, \tau\right)=1+1 /(\tau+1)$. In general, a Hausdorff measure result is much stronger than a Hausdorff dimension result as it allows one to distinguish sets of equal Hausdorff dimension. In fact, more subtle examples can be given to reiterate the significance of each of Theorems 11 and 2 For example, regarding Theorem 2. for $\tau>0$, set $\Psi_{1}(n)=\left(\beta^{n}\right)^{-\tau}$ and for some $\varepsilon>0$, set $\Psi_{\varepsilon}(n)=$ $\left(\beta^{n}\right)^{-\tau}\left(\log \left(\beta^{n}\right)\right)^{-(1+\varepsilon)(\tau+1) /(\tau+2)}$. We then have the following exact logarithmic order for $\beta$-approximation.

Corollary 1. Let $g(r)=r^{(2+\tau) /(1+\tau)}$. For any $\varepsilon>0$,

$$
\mathcal{H}^{g}\left(W\left(T_{\beta}, \Psi_{1}\right)\right)=\mathcal{H}^{g}\left([0,1]^{2}\right) \quad \text { and } \quad \mathcal{H}^{g}\left(W\left(T_{\beta}, \Psi_{\varepsilon}\right)\right)=0 .
$$

Consequently, the set $W\left(T_{\beta}, \Psi_{1}\right) \backslash W\left(T_{\beta}, \Psi_{\varepsilon}\right)$ is uncountable. 


\section{Preliminaries}

In this section, we first collect some basic properties of $\beta$-expansions and fix some notation. We then state versions of Philipp's result [28] and Beresnevich and Velani's slicing lemma 6], before giving a variant of their famous mass transference principle [5] fit for our use.

For a real number $x \geqslant 0$, we write $\lfloor x\rfloor$ for the integer part of $x$. Using the $\beta$-transformation $T_{\beta}$, each $x \in[0,1]$ can be uniquely expressed as a finite or an infinite series, known as the $\beta$-expansion of $x$; see Rényi [30]. That is, for each $x \in[0,1]$, we have

$$
x=\sum_{i \geqslant 1} \frac{\epsilon_{i}(x, \beta)}{\beta^{i}},
$$

where $\epsilon_{i}(x, \beta)=\left\lfloor\beta T_{\beta}^{i-1} x\right\rfloor$ for each $i \geqslant 1$. Now, for any $x \in[0,1]$ and $n \in \mathbb{N}$, by the definition of $\beta$-expansion,

$$
x=\frac{\epsilon_{1}(x, \beta)}{\beta}+\cdots+\frac{\epsilon_{n}(x, \beta)+T_{\beta}^{n} x}{\beta^{n}} .
$$

The $\beta$-expansion of 1 is of significant importance. To highlight this, we define an infinite sequence related to the expansion of 1 . If the expansion of 1 in (2) is infinite, that is, $\epsilon_{n}(1, \beta) \neq 0$ for infinitely many $n$, then define

$$
\left(\epsilon_{1}^{*}, \epsilon_{2}^{*}, \ldots\right)=\left(\epsilon_{1}(1, \beta), \epsilon_{2}(1, \beta), \ldots\right),
$$

and if the expansion of 1 in (2) is finite, that is

$$
1=\frac{\epsilon_{1}(1, \beta)}{\beta}+\cdots+\frac{\epsilon_{n}(1, \beta)}{\beta^{n}} \text {, with } \epsilon_{n}(1, \beta) \neq 0,
$$

then define

$$
\left(\epsilon_{1}^{*}, \epsilon_{2}^{*}, \ldots\right)=\left(\epsilon_{1}(1, \beta), \ldots, \epsilon_{n-1}(1, \beta), \epsilon_{n}(1, \beta)-1\right)^{\infty},
$$

where $w^{\infty}$ denotes the periodic sequence $(w, w, \ldots)$ for a finite word $w$. Each of the sequences $\left(\epsilon_{1}^{*}, \epsilon_{2}^{*}, \ldots\right)$ are called the infinite digit sequence of the expansion of 1 .

For each $n \in \mathbb{N}$, let $D_{\beta, n}$ denote all admissible sequences of length $n$, that is,

$$
D_{\beta, n}=\left\{\left(\epsilon_{1}, \ldots, \epsilon_{n}\right) \in \mathbb{Z}_{\geqslant 0}^{n}: \exists x \in[0,1] \text { such that } \epsilon_{i}(x, \beta)=\epsilon_{i}, 1 \leqslant i \leqslant n\right\} .
$$

The characterisation of the elements in $D_{\beta, n}$ and its cardinality $\# D_{\beta, n}$ are given by Parry [26] and Rényi [30] in the lemma below. First recall the definition of the lexicographical order $\preceq$. We write

$$
\left(\epsilon_{1}, \epsilon_{2}, \ldots, \epsilon_{n}\right) \preceq\left(\epsilon_{1}^{\prime}, \epsilon_{2}^{\prime}, \ldots, \epsilon_{n}^{\prime}\right)
$$

if for every $j \geqslant 1$ we have $\epsilon_{j} \leqslant \epsilon_{j}^{\prime}$

Lemma 1 (Parry, Rényi). A non-negative integral word $\left(\epsilon_{1}, \ldots, \epsilon_{n}\right)$ belongs to $D_{\beta, n}$ if and only if, in the lexicographical order,

$$
\left(\epsilon_{k+1}, \ldots, \epsilon_{n}\right) \preceq\left(\epsilon_{1}^{*}, \ldots, \epsilon_{n-k}^{*}\right) \text {, for all } 0 \leqslant k<n \text {. }
$$

Moreover,

$$
\beta^{n} \leqslant \# D_{\beta, n} \leqslant \frac{\beta^{n+1}}{\beta-1} .
$$

For each $\bar{\epsilon}=\left(\epsilon_{1}, \ldots, \epsilon_{n}\right) \in D_{\beta, n}$ with $n \geqslant 1$, we define the $n$th order cylinder $I_{n}(\bar{\epsilon})$ by

$$
I_{n}(\bar{\epsilon})=I_{n}\left(\epsilon_{1}, \ldots, \epsilon_{n}\right)=\left\{x \in[0,1]: \epsilon_{i}(x, \beta)=\epsilon_{i} \text { for all } 1 \leqslant i \leqslant n\right\} .
$$


The cylinder $I_{n}(\bar{\epsilon})$ is a non-empty interval with left-endpoint

$$
\frac{\epsilon_{1}}{\beta}+\cdots+\frac{\epsilon_{n}}{\beta^{n}}
$$

and with length at most $\beta^{-n}$. The exact length of a cylinder is given in [14 and it depends on the digit sequence $\epsilon_{1}, \ldots, \epsilon_{n}$.

Before moving on to results concerning various measures and sets, we note that the interval $[0,1]$ is partitioned by the cylinders $I_{n}(\bar{\epsilon})$; that is, we have the disjoint union

$$
[0,1]=\bigcup_{\bar{\epsilon} \in D_{\beta, n}} I_{n}(\bar{\epsilon})
$$

In the rest of this paper, we use the following notation concerning 'size'. For a set $A$, we denote the Lebesgue measure of $A$ by $\mathfrak{L}(A)$, and we denote the diameter of an interval $I$ by $|I|$. Of course, for an interval $I$, we have $|I|=\mathfrak{L}(I)$. Note that we also use the notation $|\cdot|$ to denote absolute value; we believe the context of usage is unambiguous. With this notation, we set out our more measure-theoretic preliminaries.

We start by recalling the following metrical result of Philipp 28] concerning the Lebesgue measure of the set $W_{y}\left(T_{\beta}, \Psi\right)$ and a one-sided variant.

Proposition 1 (Philipp). Let $W_{y}\left(T_{\beta}, \Psi\right)$ be the set defined in (11), and define

$$
W_{y}^{\prime}\left(T_{\beta}, \Psi\right):=\left\{x \in[0,1]: 0 \leqslant T_{\beta}^{n} x-y<\Psi(n) \text { for infinitely many } n\right\} .
$$

Then

$$
\mathfrak{L}\left(W_{y}\left(T_{\beta}, \Psi\right)\right)=\mathfrak{L}\left(W_{y}^{\prime}\left(T_{\beta}, \Psi\right)\right)=\left\{\begin{array}{l}
0 \text { when } \sum_{n \geqslant 1} \Psi(n) \text { converges } \\
1 \quad \text { when } \sum_{n \geqslant 1} \Psi(n) \text { diverges. }
\end{array}\right.
$$

While both parts of Proposition 1 are special cases of Philipp's result [28, as a sequence of intervals takes on the role of the balls $\{B(y, \Psi(n))\}_{n \geqslant 1}$, it is worth noting that the result for $W_{y}^{\prime}\left(T_{\beta}, \Psi\right)$ can also be deduced from the result for $W_{y}\left(T_{\beta}, \Psi\right)$ using the Lebesgue density theorem. We cite a general result due to Cassels; see Harman [16, Lemma 2.1].

Lemma 2 (Cassels). Let $\left\{I_{k}\right\}_{k \geqslant 1}$ be a sequence of intervals such that $\mathfrak{L}\left(I_{k}\right) \rightarrow 0$ as $k \rightarrow \infty$. If $\left\{J_{k}\right\}_{k \geqslant 1}$ is a sequence of measurable sets such that $J_{k} \subseteq I_{k}$ for each $k \geqslant 1$, and there is a positive real number $\delta$ such that $\mathfrak{L}\left(J_{k}\right) \geqslant \delta \cdot \mathfrak{L}\left(I_{k}\right)$, then

$$
\mathfrak{L}\left(\limsup _{k \rightarrow \infty} J_{k}\right)=\mathfrak{L}\left(\limsup _{k \rightarrow \infty} I_{k}\right) .
$$

We next state a variant of the 'slicing' lemma due to Beresnevich and Velani [6]. This version is tailored for our use, and is a key ingredient in the proof of Theorem 2. The slicing technique is broad-ranging and has been useful in proving several metrical results; for examples, see Hussain and Kristensen [21, 22] and Hussain and Levesley [23].

Lemma 3 (Beresnevich and Velani). Suppose that $g$ and $f: r \rightarrow r^{-1} g(r)$ are dimension functions. Let $B \subseteq \mathbb{R}^{2}$ be a Borel set and let $V$ be a 1-dimensional linear subspace of $\mathbb{R}^{2}$. If there is a subset $S$ of the orthogonal complement of $V$ such that $\mathcal{H}^{1}(S)>0$ and for each $b \in S$,

$$
\mathcal{H}^{f}(B \cap(V+b))=\infty,
$$

then $\mathcal{H}^{g}(B)=\infty$. 
The main ingredient in establishing Theorem 1 is the mass transference principle of Beresnevich and Velani [5]. Given a dimension function $f$ and a sequence of balls $B_{i} \subseteq \mathbb{R}$, by definition, $\lim _{\sup _{i \rightarrow \infty}} B_{i}$ is precisely the set of points which lie in infinitely many of the balls $B_{i}$. Further, for a ball $B=B(x, r)$, set $B^{f}=B(x, f(r))$. The following mass transference principle is tailored to suit our needs; for a general statement and further details, we refer the reader to the paper of Beresnevich and Velani [5, Theorem 2].

Proposition 2 (Mass Transference Principle). Let $\left\{B_{i}\right\}_{i \geqslant 1}$ be a sequence of balls in $\mathbb{R}$ with $\left|B_{i}\right| \rightarrow 0$ as $i \rightarrow \infty$ and let $f$ be a dimension function such that $r^{-1} f(r)$ is non-decreasing as $r \rightarrow 0$. Suppose that for any ball $B \subseteq \mathbb{R}$

$$
\mathcal{H}^{1}\left(B \cap \lim \sup B_{i}^{f}\right)=\mathcal{H}^{1}(B) .
$$

Then for any ball $B \subseteq \mathbb{R}$,

$$
\mathcal{H}^{f}\left(B \cap \limsup B_{i}\right)=\mathcal{H}^{f}(B) .
$$

In essence, the mass transference principle allows one to translate statements about the Lebesgue measure of general limsup sets to ones involving Hausdorff measure. So, using Proposition 1 one should be able to say something about the Hausdorff measure of $W_{y}\left(T_{\beta}, \Psi\right)$. Indeed, this turns out to be the case, but we must first make some minor modifications to the mass transference principle.

Proposition 3 (A variant of Mass Transference Principle). Let $\left\{x_{n}\right\}_{n \geqslant 1}$ be a sequence of points in $[0,1]$ and $\left\{r_{n}\right\}_{n \geqslant 1}$ a sequence of positive numbers with $r_{n} \rightarrow 0$ as $n \rightarrow \infty$. Let $f$ be a dimension function such that $r^{-1} f(r)$ is non-decreasing as $r \rightarrow 0$. If

$$
\mathfrak{L}\left(\left\{x \in[0,1]: 0 \leqslant x-x_{n}<f\left(r_{n}\right) \text { for infinitely many } n\right\}\right)=1,
$$

then for any ball $B \subseteq \mathbb{R}$,

$$
\mathcal{H}^{f}\left(B \cap\left\{x \in[0,1]: 0 \leqslant x-x_{n}<r_{n} \text { for infinitely many } n\right\}\right)=\infty .
$$

With the use of a tiny variant of the $K_{G, B}$ Lemma [5, Lemma 5], our variant of the mass transference principle is proved, mutatis mutandis, as Proposition 2 (see Beresnevich and Velani [5], Theorem 2]), thus we only present a variant of the $K_{G, B}$ Lemma.

For a subset $\mathcal{K} \subseteq\left\{\left[x_{n}, x_{n}+r_{n}\right): n \geqslant 1\right\}$, we define

$$
\mathcal{K}^{f}:=\left\{B^{f}\left(x_{n}, r_{n}\right):\left[x_{n}, x_{n}+r_{n}\right) \in \mathcal{K}\right\},
$$

where $B^{f}\left(x_{n}, r_{n}\right)$ denotes the ball of radius $f\left(r_{n}\right)$ centred at $x_{n}$.

Lemma 4 (A variant of $K_{G, B}$ Lemma). Assume that the equation in (6) holds and let $B$ be a ball in $[0,1]$. For any $G \geqslant 1$, there exists a subset $K_{G, B} \subseteq\left\{\left[x_{n}, x_{n}+\right.\right.$ $\left.\left.r_{n}\right)\right\}_{n \geqslant G}$ such that the elements of $K_{G, B}^{f}$ are disjoint, inside $B$ and

$$
\sum_{L \in K_{G}, B} f\left(r_{L}\right) \geqslant \frac{|B|}{20},
$$

where $r_{L}$ denotes the radius of the ball $L$.

Proof. The elements in $K_{G, B}$ here are nothing but half of the balls in Beresnevich and Velani's original $K_{G, B}$-Lemma [5, Lemma 5].

Remark 1. Note that while we state our variant of the mass transference principle only in the one-dimensional case, it is still valid for higher dimensions. 


\section{Dichotomy LAWS FOR $\beta$-DYNAMiCAL SyStems}

In this section, we establish the Jarník-type dichotomy laws of Theorems 1 and 2 .

Proof of Theorem 1. We consider, separately, the cases of convergence and divergence of the series

$$
\sum_{n \geqslant 1} \beta^{n} f\left(\frac{\Psi(n)}{\beta^{n}}\right)
$$

Suppose that the series (17) converges. We begin by writing the set $W_{y}\left(T_{\beta}, \Psi\right)$ in a way that reflects its limsup nature. To do this, for any $\bar{\epsilon}=\left(\epsilon_{1}, \ldots, \epsilon_{n}\right) \in D_{\beta, n}$, we define

$$
y_{n}(\bar{\epsilon})=\frac{\epsilon_{1}}{\beta}+\cdots+\frac{\epsilon_{n}}{\beta^{n}}+\frac{y}{\beta^{n}} .
$$

We then have

$$
\begin{aligned}
W_{y}\left(T_{\beta}, \Psi\right) & =\bigcap_{N \geqslant 1} \bigcup_{n \geqslant N}\left\{x \in[0,1]:\left|T_{\beta}^{n} x-y\right|<\Psi(n)\right\} \\
& =\bigcap_{N \geqslant 1} \bigcup_{\substack{n \geqslant N \\
\bar{\epsilon} \in D_{\beta, n}}}\left\{x \in I_{n}(\bar{\epsilon}):\left|T_{\beta}^{n} x-y\right|<\Psi(n)\right\} \\
& =\bigcap_{N \geqslant 1} \bigcup_{\substack{n \geqslant N \\
\bar{\epsilon} \in D_{\beta, n}}}\left\{I_{n}(\bar{\epsilon}) \cap\left(y_{n}(\bar{\epsilon})-\frac{\Psi(n)}{\beta^{n}}, y_{n}(\bar{\epsilon})+\frac{\Psi(n)}{\beta^{n}}\right)\right\},
\end{aligned}
$$

where, to obtain the last equality, we substituted the value of $T_{\beta}^{n} x$ in terms of $\bar{\epsilon}$, which is determined by (3). Note that the set inside the union in (9) can be covered by two intervals each of length $\frac{\Psi(n)}{\beta^{n}}$, thus along with the definition of Hausdorff measure, the quantity $\mathcal{H}^{f}\left(W_{y}\left(T_{\beta}, \Psi\right)\right)$ is bounded by

$$
\liminf _{N \rightarrow \infty} \sum_{n \geqslant N} \sum_{\bar{\epsilon} \in D_{\beta, n}} 2 \cdot f\left(\frac{\Psi(n)}{\beta^{n}}\right) \leqslant \frac{2 \beta}{\beta-1} \liminf _{N \rightarrow \infty} \sum_{n \geqslant N} \beta^{n} f\left(\frac{\Psi(n)}{\beta^{n}}\right)=0,
$$

where for the second inequality, we used $\# D_{\beta, n} \leqslant \beta^{n+1} /(\beta-1)$ as given in (4).

Now suppose that the series (7) diverges. If $\{f(r) / r: r>0\}$ is bounded, $\mathcal{H}^{f}=c \cdot \mathfrak{L}$ for a constant $c>0$. Then Philipp's result applies. So, we assume that $f(r) / r \rightarrow \infty$ as $r \rightarrow 0$. Moreover, instead of studying the set $W_{y}\left(T_{\beta}, \Psi\right)$ directly, we consider the sets $W_{y}^{\prime}\left(T_{\beta}, \Psi\right)$ (as defined in Proposition 10) and

$W_{y}^{\prime}\left(T_{\beta}, \Psi, f\right)=\left\{x \in[0,1]: 0 \leqslant T_{\beta}^{n} x-y<\beta^{n} f\left(\frac{\Psi(n)}{\beta^{n}}\right)\right.$ for infinitely many $\left.n\right\}$.

Because of our divergence assumption on the series (7), by Proposition 1] we have $\mathfrak{L}\left(W_{y}^{\prime}\left(T_{\beta}, \Psi, f\right)\right)=1$.

Similar to the above, we may write

$$
W_{y}^{\prime}\left(T_{\beta}, \Psi\right)=\bigcap_{N \geqslant 1} \bigcup_{\substack{n \geqslant N \\ \bar{\epsilon} \in D_{\beta, n}}}\left\{I_{n}(\bar{\epsilon}) \cap\left[y_{n}(\bar{\epsilon}), y_{n}(\bar{\epsilon})+\frac{\Psi(n)}{\beta^{n}}\right)\right\}
$$

and

$$
W_{y}^{\prime}\left(T_{\beta}, \Psi, f\right):=\bigcap_{N \geqslant 1} \bigcup_{\substack{n \geqslant N \\ \bar{\epsilon} \in D_{\beta, n}}}\left\{I_{n}(\bar{\epsilon}) \cap\left[y_{n}(\bar{\epsilon}), y_{n}(\bar{\epsilon})+f\left(\frac{\Psi(n)}{\beta^{n}}\right)\right)\right\}
$$

where $y_{n}(\bar{\epsilon})$ is as given in (8). 
Since $y_{n}(\bar{\epsilon})$ is larger than the left endpoint of $I_{n}(\bar{\epsilon})$, we have

$$
\begin{aligned}
I_{n}(\bar{\epsilon}) \cap\left[y_{n}(\bar{\epsilon}), y_{n}(\bar{\epsilon})+\frac{\Psi(n)}{\beta^{n}}\right) & =\left\{x \in[0,1]: 0 \leqslant x-y_{n}(\bar{\epsilon})<r_{n}(\bar{\epsilon})\right\} \\
& =\left[y_{n}(\bar{\epsilon}), y_{n}(\bar{\epsilon})+r_{n}(\bar{\epsilon})\right)
\end{aligned}
$$

for some $r_{n}(\bar{\epsilon}) \geqslant 0$ (we take $r_{n}(\bar{\epsilon})=0$ if the set is empty). So,

$$
W_{y}^{\prime}\left(T_{\beta}, \Psi\right)=\bigcap_{N \geqslant 1} \bigcup_{\substack{n \geqslant N \\ \bar{\epsilon} \in D_{\beta, n}}}\left\{x \in[0,1]: 0 \leqslant x-y_{n}(\bar{\epsilon})<r_{n}(\bar{\epsilon})\right\} .
$$

Similarly,

$$
\begin{aligned}
I_{n}(\bar{\epsilon}) \cap\left[y_{n}(\bar{\epsilon}), y_{n}(\bar{\epsilon})+f\left(\frac{\Psi(n)}{\beta^{n}}\right)\right) & =\left\{x \in[0,1]: 0 \leqslant x-y_{n}(\bar{\epsilon})<t_{n}(\bar{\epsilon})\right\} \\
& =\left[y_{n}(\bar{\epsilon}), y_{n}(\bar{\epsilon})+t_{n}(\bar{\epsilon})\right)
\end{aligned}
$$

for some $t_{n}(\bar{\epsilon}) \geqslant 0$ (we take $t_{n}(\bar{\epsilon})=0$ if the set is empty), and so

$$
W_{y}^{\prime}\left(T_{\beta}, \Psi, f\right)=\bigcap_{N \geqslant 1} \bigcup_{\substack{n \geqslant N \\ \bar{\epsilon} \in D_{\beta, n}}}\left\{x \in[0,1]: 0 \leqslant x-y_{n}(\bar{\epsilon})<t_{n}(\bar{\epsilon})\right\} .
$$

We claim that for sufficiently large $n$,

$$
f\left(r_{n}(\bar{\epsilon})\right) \geqslant t_{n}(\bar{\epsilon})
$$

for any $\bar{\epsilon} \in D_{\beta, n}$. To see that this is indeed the case, let $b$ be the right endpoint of $I_{n}(\bar{\epsilon})$. Then

$$
y_{n}(\bar{\epsilon})+r_{n}(\bar{\epsilon})=\min \left\{b, y_{n}(\bar{\epsilon})+\frac{\Psi(n)}{\beta^{n}}\right\}
$$

and

so that

$$
y_{n}(\bar{\epsilon})+t_{n}(\bar{\epsilon})=\min \left\{b, y_{n}(\bar{\epsilon})+f\left(\frac{\Psi(n)}{\beta^{n}}\right)\right\} \text {, }
$$

$$
r_{n}(\bar{\epsilon})=\min \left\{b-y_{n}(\bar{\epsilon}), \frac{\Psi(n)}{\beta^{n}}\right\} \quad \text { and } \quad t_{n}(\bar{\epsilon})=\min \left\{b-y_{n}(\bar{\epsilon}), f\left(\frac{\Psi(n)}{\beta^{n}}\right)\right\} .
$$

Now if $t_{n}(\bar{\epsilon})=0$, there is nothing to prove. When $t_{n}(\bar{\epsilon})>0$, we have $y_{n}(\bar{\epsilon})<b$, thus we have $r_{n}(\bar{\epsilon})>0$ as well. Since $r^{-1} f(r) \rightarrow \infty$ as $r \rightarrow 0$ and $0 \leqslant b-y_{n}(\bar{\epsilon}) \leqslant \beta^{-n}$, we have that $f\left(b-y_{n}(\bar{\epsilon})\right) \geqslant b-y_{n}(\bar{\epsilon})$ when $n$ is sufficiently large, which proves the claim. Note also that $0 \leqslant r_{n}(\bar{\epsilon}) \leqslant \beta^{-n}$, so that $r_{n}(\bar{\epsilon}) \rightarrow 0$ as $n \rightarrow \infty$.

Having established (11), the limsup set

$$
\bigcap_{N \geqslant 1} \bigcup_{\substack{n \geqslant N \\ \bar{\epsilon} \in D_{\beta, n}}}\left\{x \in[0,1]: 0 \leqslant x-y_{n}(\bar{\epsilon})<f\left(r_{n}(\bar{\epsilon})\right)\right\}
$$

contains the set $W_{y}^{\prime}\left(T_{\beta}, \Psi, f\right)$, so it is also of full Lebesgue measure. Then a direct application of our variant of the mass transference principle (Proposition 3) yields $\mathcal{H}^{f}\left(W_{y}^{\prime}\left(T_{\beta}, \Psi\right)\right)=\infty$. As $W_{y}^{\prime}\left(T_{\beta}, \Psi\right) \subseteq W_{y}\left(T_{\beta}, \Psi\right)$, the result follows.

Remark 2. Loosely speaking, $y_{n}(\bar{\epsilon})$ is the inverse $T_{\beta}^{-n} y$ of $y$ in the cylinder $I_{n}(\bar{\epsilon})$. But, this is not always the case. When the length of the interval $I_{n}(\bar{\epsilon})$ is strictly less than $\beta^{-n}$, for any $y>\beta^{n}\left|I_{n}(\bar{\epsilon})\right|$, there does not exist $x \in I_{n}(\bar{\epsilon})$, such that $T_{\beta}^{n} x=y$. So, it is possible that

$$
I_{n}(\bar{\epsilon}) \cap\left(y_{n}(\bar{\epsilon})-\frac{\Psi(n)}{\beta^{n}}, y_{n}(\bar{\epsilon})+\frac{\Psi(n)}{\beta^{n}}\right)
$$


is empty. In view of this, the magnified set

$$
I_{n}(\bar{\epsilon}) \cap\left(y_{n}(\bar{\epsilon})-f\left(\frac{\Psi(n)}{\beta^{n}}\right), y_{n}(\bar{\epsilon})+f\left(\frac{\Psi(n)}{\beta^{n}}\right)\right)
$$

may contribute to the Lebesgue measure of the limsup set

$$
\left\{x \in[0,1]:\left|T_{\beta}^{n} x-y\right|<\beta^{n} f\left(\frac{\Psi(n)}{\beta^{n}}\right) \text { for infinitely many } n\right\},
$$

while the shrunk set

$$
I_{n}(\bar{\epsilon}) \cap\left(y_{n}(\bar{\epsilon})-\frac{\Psi(n)}{\beta^{n}}, y_{n}(\bar{\epsilon})+\frac{\Psi(n)}{\beta^{n}}\right)
$$

may contribute nothing to the size of $W_{y}(T, \Psi)$. This is the main reason we consider the subset $W_{y}^{\prime}(T, \Psi)$, instead of $W_{y}(T, \Psi)$.

We continue this section with the proof of Theorem 2 .

Proof of Theorem 2. We consider, separately, the cases of convergence and divergence of the series

$$
\sum_{n \geqslant 1} g\left(\frac{\Psi(n)}{\beta^{n}}\right) \frac{\beta^{2 n}}{\Psi(n)}
$$

As in the proof of Theorem 1 we begin by writing the limsup version of the set $W\left(T_{\beta}, \Psi\right)$. To do this, for each $n \in \mathbb{N}$, set

$$
W_{n}\left(T_{\beta}, \Psi\right):=\left\{(x, y) \in[0,1]^{2}:\left|T_{\beta}^{n} x-y\right|<\Psi(n)\right\} .
$$

Then

$$
W\left(T_{\beta}, \Psi\right)=\limsup _{n \rightarrow \infty} W_{n}\left(T_{\beta}, \Psi\right)=\bigcap_{N \geqslant 1} \bigcup_{n \geqslant N} W_{n}\left(T_{\beta}, \Psi\right) .
$$

On the other hand, the interval $[0,1]$, which corresponds to the doubly metric parameter $y$, can also be written as a union of intervals

$$
J_{n}(i)=\left[\frac{i \Psi(n)}{\beta^{n}}, \frac{(i+1) \Psi(n)}{\beta^{n}}\right] \cap[0,1]
$$

over all $0 \leqslant i \leqslant\left\lfloor\beta^{n} / \Psi(n)\right\rfloor$; that is,

$$
[0,1]=\bigcup_{0 \leqslant i \leqslant\left\lfloor\beta^{n} / \Psi(n)\right\rfloor} J_{n}(i) .
$$

Combining (5) and (15), we have

$$
[0,1]^{2}=\bigcup_{\substack{\bar{\epsilon} \in D_{\beta, n} \\ 0 \leqslant i \leqslant\left\lfloor\beta^{n} / \Psi(n)\right\rfloor}} I_{n}(\bar{\epsilon}) \times J_{n}(i) .
$$

This, when combined with (14), gives

$$
W_{n}\left(T_{\beta}, \Psi\right)=\bigcup_{\substack{\bar{\epsilon} \in D_{\beta, n} \\ 0 \leqslant i \leqslant\left\lfloor\beta^{n} / \Psi(n)\right\rfloor}}\left\{(x, y) \in I_{n}(\bar{\epsilon}) \times J_{n}(i):\left|T_{\beta}^{n} x-y\right|<\Psi(n)\right\} .
$$

Note that, given any $\bar{\epsilon} \in D_{\beta, n}$ and $0 \leqslant i \leqslant\left\lfloor\beta^{n} / \Psi(n)\right\rfloor$, if

$$
(x, y) \in\left\{I_{n}(\bar{\epsilon}) \times J_{n}(i):\left|T_{\beta}^{n} x-y\right|<\Psi(n)\right\}
$$

then

$$
\left|T_{\beta}^{n} x-\frac{i \Psi(n)}{\beta^{n}}\right| \leqslant\left|T_{\beta}^{n} x-y\right|+\left|y-\frac{i \Psi(n)}{\beta^{n}}\right| \leqslant \Psi(n)+\frac{\Psi(n)}{\beta^{n}}<2 \Psi(n) .
$$

Set

$$
z_{n}=\frac{\epsilon_{1}}{\beta}+\cdots+\frac{\epsilon_{n}}{\beta^{n}}+\frac{i \Psi(n)}{\beta^{n}} .
$$


Then, similar to the convergence part of the proof of Theorem 1, it follows that

$$
x \in\left(z_{n}-\frac{2 \Psi(n)}{\beta^{n}}, z_{n}+\frac{2 \Psi(n)}{\beta^{n}}\right) .
$$

Thus

$$
W_{n}\left(T_{\beta}, \Psi\right) \subseteq \bigcup_{\substack{\epsilon \in D_{\beta}, n \\ 0 \leqslant i \leqslant\left\lfloor\beta^{n} / \Psi(n)\right\rfloor}}\left(z_{n}-\frac{2 \Psi(n)}{\beta^{n}}, z_{n}+\frac{2 \Psi(n)}{\beta^{n}}\right) \times J_{n}(i) .
$$

As a result, for $N \geqslant 1$, the family of rectangles

$$
\bigcup_{\substack{n \geqslant N \\ \bar{\epsilon} \in D_{\beta, n} \\ i \leqslant\left\lfloor\beta^{n} / \Psi(n)\right\rfloor}}\left(z_{n}-\frac{2 \Psi(n)}{\beta^{n}}, z_{n}+\frac{2 \Psi(n)}{\beta^{n}}\right) \times J_{n}(i)
$$

is a cover for the set $W_{n}\left(T_{\beta}, \Psi\right)$. Moreover, each of these rectangles can be covered by 64 cubes with diameter $\Psi(n) / \beta^{n}$. So, by the definition of Hausdorff measure, it follows, using (4), that $\mathcal{H}^{g}\left(W\left(T_{\beta}, \Psi\right)\right)$ is bounded by a constant times

$$
\liminf _{N \rightarrow \infty} \sum_{n \geqslant N} \sum_{\bar{\epsilon} \in D_{\beta, n}} \sum_{0 \leqslant i \leqslant\left\lfloor\beta^{n} / \Psi(n)\right\rfloor} g\left(\frac{\Psi(n)}{\beta^{n}}\right) \ll \liminf _{N \rightarrow \infty} \sum_{n \geqslant N} g\left(\frac{\Psi(n)}{\beta^{n}}\right) \frac{\beta^{2 n}}{\Psi(n)} .
$$

If the series in (13) converges, it follows from (16) that $\mathcal{H}^{g}\left(W\left(T_{\beta}, \Psi\right)\right)=0$.

Now suppose that the series in (13) diverges, and set $f(x)=x^{-1} g(x)$. Then

$$
\sum_{n \geqslant 1} f\left(\frac{\Psi(n)}{\beta^{n}}\right) \beta^{n}=\sum_{n \geqslant 1} g\left(\frac{\Psi(n)}{\beta^{n}}\right) \frac{\beta^{2 n}}{\Psi(n)}=\infty .
$$

By Theorem 11, we have shown that for any fixed $y \in[0,1], \mathcal{H}^{f}\left(W_{y}\left(T_{\beta}, \Psi\right)\right)=$ $\mathcal{H}^{f}([0,1])$. Then, appealing to the slicing lemma (Lemma 3]), we have immediately that $\mathcal{H}^{g}\left(W\left(T_{\beta}, \Psi\right)\right)=\mathcal{H}^{g}\left([0,1]^{2}\right)$.

\section{Concluding Remarks And Questions}

In this paper, we considered the $\beta$-expansion of real numbers for real values of $\beta>1$. Of course, it is very interesting to restrict $\beta$ to the set of algebraic numbers, and even more interesting to restrict both $\beta$ and the numbers we are expanding to be algebraic numbers. To the best of our knowledge, the only known consideration of this case was made by Bugeaud 8 , who gave results concerning the number of digit changes in the $\beta$-expansion of algebraic numbers (where $\beta$ is algebraic). The questions of Hausdorff dimension and Hausdorff measure, as well as the possibility of any dichotomy law, are still open.

As we mentioned in the Introduction, the set $W_{y}\left(T_{\beta}, \Psi\right)$ is closely related to the distribution of the preimage

$$
\operatorname{Pre}\left(T_{\beta}, y\right)=\left\{T_{\beta}^{-n} y, n \in \mathbb{N}\right\}
$$

of $y$. Given a general compact metric space $(X, T)$, one can consider the size of

$$
W(T, \Psi)=\left\{x \in X:\left|T^{n} x-y\right|<\Psi(n) \text { for infinitely many } n\right\} .
$$

If $\operatorname{Pre}(T, y)$ is well-distributed, one hopes to derive some information on the Hausdorff dimension and Hausdorff measure of $W(T, \Psi)$. But how well should Pre $(T, y)$ be distributed sufficient to get the dimension or even measure? Can one give a precise characterisation on this requirement? To which extent can a general system fulfill the required conditions? 
Acknowledgements. MH would like to thank Stephen Harrap for many useful discussions and to members of the Departmetn of Mathematics at Huazhong University for their hospitality.

\section{REFERENCES}

1. D. Badziahin, V. Beresnevich, and S. Velani, Inhomogeneous theory of dual Diophantine approximation on manifolds, Adv. Math. 232 (2013), 1-35. MR 2989975

2. D. Badziahin, S. Harrap, and M. Hussain, An inhomogeneous Jarnik type theorem for planar curves, arXiv:1503.04981 (2015), preprint.

3. A. Baker and W. Schmidt, Diophantine approximation and Hausdorff dimension, Proc. London Math. Soc. (3) 21 (1970), 1-11. MR 0271033 (42 \#5916)

4. - Measure theoretic laws for lim sup sets, Mem. Amer. Math. Soc. 179 (2006), no. 846, $\mathrm{x}+91$. MR 2184760 (2007d:11086)

5. V. Beresnevich and S. Velani, A mass transference principle and the Duffin-Schaeffer conjecture for Hausdorff measures, Ann. of Math. (2) 164 (2006), no. 3, 971-992. MR 2259250 (2008a:11090)

6. , Schmidt's theorem, Hausdorff measures, and slicing, Int. Math. Res. Not. (2006), Art. ID 48794, 24. MR 2264714 (2007h:11090)

7. V. I. Bernik and M. M. Dodson, Metric Diophantine approximation on manifolds, Cambridge Tracts in Mathematics, vol. 137, Cambridge University Press, Cambridge, 1999. MR 1727177 (2001h:11091)

8. Y. Bugeaud, On the $\beta$-expansion of an algebraic number in an algebraic base $\beta$, Integers 9 (2009), A20, 215-226. MR 2534910 (2010i:11101)

9. Y. Bugeaud and B. Wang, Distribution of full cylinders and the Diophantine properties of the orbits in $\beta$-expansions, J. Fractal Geom. 1 (2014), no. 2, 221-241. MR 3230505

10. N. Chernov and D. Kleinbock, Dynamical Borel-Cantelli lemmas for Gibbs measures, Israel J. Math. 122 (2001), 1-27. MR 1826488 (2002h:37003)

11. M. M. Dodson, A note on metric inhomogeneous Diophantine approximation, J. Austral. Math. Soc. Ser. A 62 (1997), no. 2, 175-185. MR 1433207 (98b:11085)

12. M. M. Dodson, B. P. Rynne, and J. A. G. Vickers, Diophantine approximation and a lower bound for Hausdorff dimension, Mathematika 37 (1990), no. 1, 59-73. MR 1067887 (91i:11098)

13. K. Falconer, Fractal geometry, second ed., John Wiley \& Sons, Inc., Hoboken, NJ, 2003, Mathematical foundations and applications. MR 2118797 (2006b:28001)

14. A.-H. Fan and B. Wang, On the lengths of basic intervals in beta expansions, Nonlinearity $\mathbf{2 5}$ (2012), no. 5, 1329-1343. MR 2914142

15. Yuehua Ge and Fan Lü, A note on inhomogeneous Diophantine approximation in betadynamical system, Bull. Aust. Math. Soc. 91 (2015), no. 1, 34-40. MR 3294256

16. G. Harman, Metric number theory, London Mathematical Society Monographs. New Series, vol. 18, The Clarendon Press, Oxford University Press, New York, 1998. MR 1672558 (99k:11112)

17. R. Hill and S. Velani, The ergodic theory of shrinking targets, Invent. Math. 119 (1995), no. 1, 175-198. MR 1309976 (96e:58088)

18. _ The shrinking target problem for matrix transformations of tori, J. London Math. Soc. (2) 60 (1999), no. 2, 381-398. MR 1724857 (2000i:37003)

19. A zero-infinity law for well-approximable points in Julia sets, Ergodic Theory Dynam. Systems 22 (2002), no. 6, 1773-1782. MR 1944403 (2003m:37065)

20. F. Hofbauer, $\beta$-shifts have unique maximal measure, Monatsh. Math. 85 (1978), no. 3, 189198. MR 0492180 (58 \#11326)

21. M. Hussain and S. Kristensen, Metrical results on systems of small linear forms, Int. J. Number Theory 9 (2013), no. 3, 769-782. MR 3043613

22. (2015), preprint.

23. M. Hussain and J. Levesley, The metrical theory of simultaneously small linear forms, Funct. Approx. Comment. Math. 48 (2013), no. part 2, 167-181. MR 3100138

24. M. Fuchs and D. Kim, On Kurzweil's 0-1 Law in Inhomogeneous Diophantine Approximation, arXiv:1501.04714, 2015.

25. B. Li, B. Wang, J. Wu, and J. Xu, The shrinking target problem in the dynamical system of continued fractions, Proc. Lond. Math. Soc. (3) 108 (2014), no. 1, 159-186. MR 3162824

26. W. Parry, On the $\beta$-expansions of real numbers, Acta Math. Acad. Sci. Hungar. 11 (1960), 401-416. MR 0142719 (26 \#288) 
27. T. Persson and J. Schmeling, Dyadic Diophantine approximation and Katok's horseshoe approximation, Acta Arith. 132 (2008), no. 3, 205-230. MR 2403650 (2009c:11111)

28. W. Philipp, Some metrical theorems in number theory, Pacific J. Math. 20 (1967), 109-127. MR 0205930 (34 \#5755)

29. H. Reeve, Shrinking targets for countable Markov maps, arXiv:1107.4736 (2011), preprint.

30. A. Rényi, Representations for real numbers and their ergodic properties, Acta Math. Acad. Sci. Hungar 8 (1957), 477-493. MR 0097374 (20 \#3843)

31. J. Schmeling, Symbolic dynamics for $\beta$-shifts and self-normal numbers, Ergodic Theory Dynam. Systems 17 (1997), no. 3, 675-694. MR 1452189 (98c:11080)

32. L. Shen and B. Wang, Shrinking target problems for beta-dynamical system, Sci. China Math. 56 (2013), no. 1, 91-104. MR 3016585

33. B. Tan and B. Wang, Quantitative recurrence properties for beta-dynamical system, Adv. Math. 228 (2011), no. 4, 2071-2097. MR 2836114

34. J. Tseng, On circle rotations and the shrinking target properties, Discrete Contin. Dyn. Syst. 20 (2008), no. 4, 1111-1122. MR 2379490 (2010a:37080)

35. M. Urbański, Diophantine analysis of conformal iterated function systems, Monatsh. Math. 137 (2002), no. 4, 325-340. MR 1947918 (2004j:37085)

School of Mathematical and Physical Sciences, University of Newcastle, Callaghan, 2308, NSW, Australia

E-mail address: michael.coons@newcastle.edu.au

School of Mathematical and Physical Sciences, University of Newcastle, Callaghan, 2308, NSW, Australia

E-mail address: mumtaz.hussain@newcastle.edu.au, drhussainmumtaz@gmail.com

School of Mathematics and Statistics, Huazhong University of Science and TechNOLOGY, 430074 WuHAn, ChinA

E-mail address: bwei_wang@hust.edu.cn 